\title{
Estimating MIMO System Performance Using the Correlation Matrix Approach
}

\author{
Sergey Loyka, Member, IEEE, and George Tsoulos, Member, IEEE
}

\begin{abstract}
The channel capacity of a multiple-input-multipleoutput (MIMO) communication system depends substantially on correlation between individual receive branches. In this letter, we investigate the MIMO capacity using the correlation matrix approach and the Salz-Winters spatial correlation model. It is shown that for a linear array, correlation has no impact on the MIMO channel capacity provided that the two-element array beamwidth is smaller than the angle spread of the incoming signals. Simple but accurate approximations for the correlation coefficient and the corresponding channel capacity are derived for different angular spreads.
\end{abstract}

Index Terms-Channel capacity, fading correlation, MIMO.

\section{INTRODUCTION}

$\mathbf{M}$ IMO communication architectures provide substantially better spectral efficiency than traditional systems under certain conditions [1]. However, the electromagnetic environment has a profound impact on their operation. In particular, correlation between individual sub-channels of the matrix channel may result in severe degradation of the MIMO architecture performance. Several models have been considered for the estimation of this effect [2]-[4]. In this letter, the correlation matrix approach [3], [5], [6] is employed together with the Salz-Winters model of the fading spatial correlation [8] in order to predict the MIMO architecture performance in a realistic electromagnetic environment. This also allows to obtain an explicit relationship between MIMO channel capacity and the average angle of arrival and angle spread of incoming signals.

\section{CORRElation Matrix MODEL}

We employ here the spatial correlation model presented in [8]. In this model, each user generates many independent multipath signals arriving to the adaptive array within $\pm \Delta$ of the mean angle of arrival (AOA) $\varphi$ (see Fig. 1). The AOA probability density function is assumed to be uniform and all users are assumed to be statistically independent and to have the same

Manuscript received May 31, 2001. The associate editor coordinating the review of this letter and approving it for publication was Prof. K. Kiasaleh.

S. Loyka is with the School of Information Technology and Engineering (SITE), University of Ottawa, Ottawa, ON, Canada, K1N 6N5 (e-mail sergey.loyka@ieee.org).

G. Tsoulos is with Cambridge Technology Centre, Wireless Technology Melbourn, Hertfordshire, SG8 6DP, U.K. (e-mail: george.tsoulos@pa-consulting.com).

Publisher Item Identifier S 1089-7798(02)00974-2.

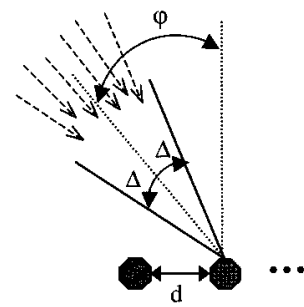

Fig. 1. Incoming multipath signals arrive to the linear antenna array within $\pm \Delta$ of mean angle $\varphi$.

statistics. The signal correlation coefficient between the $i$ th and $k$ th antenna array elements is

$$
R_{i k}=\frac{1}{2 \Delta} \int_{\varphi-\Delta}^{\varphi+\Delta} \exp [j z(i-k) \sin \beta] d \beta
$$

where $z=2 \pi d / \lambda, d$ is the inter-element distance, $\lambda$ is the wavelength, $2 \Delta$ is the angle spread of the incoming multipaths, $\varphi$ is the average angle of arrival, and $j$ is the imaginary unit. Without loss of generality, we assume that $\lambda=1$. For $\Delta=\pi$, (1) reduces to the classical expression [9]

$$
R_{i k}=J_{0}[z(i-k)]
$$

where $J_{0}$ is the zeroth-order Bessel function of the first kind. For $\Delta<\pi$ a Bessel series expansion was derived for $R_{i k}$ in [8]. However, as detailed analysis shows, for small $\Delta$ (a few degrees) this expansion converges very slowly and, consequently, a large number of terms must be used in order to estimate $R_{i k}$ accurately. The computational efficiency of this procedure is very low, especially when matrix computations are involved, as is the case for MIMO systems. A simple but still accurate approximation of (1) for small $\Delta$ and $\varphi=0$ can be derived using $\sin \beta \approx \beta$ (valid for small $\beta$ ), and performing integration in (1):

$$
R_{i k} \approx \frac{\sin z(i-k) \Delta}{z(i-k) \Delta}
$$

The smaller $\Delta$, the better the accuracy is. Thus, this approximation works exactly where it is needed. The upper bound of its validity is approximately $\pi / 4$. Hence, one may use (2) for large values of $\Delta$ and (3) for small values.

\section{MIMO CHANNEL CAPACITY}

For fixed linear $n \times n$ matrix channel with additive white Gaussian noise and when the transmitted signal vector is composed of statistically independent equal power components each with a Gaussian distribution, the channel capacity is [1]

$$
C=\log _{2} \operatorname{det}\left(\mathbf{I}+\frac{\rho}{n} \mathbf{H} \cdot \mathbf{H}^{+}\right) \text {bits/s/Hz }
$$


where $n$ is the number of transmit/receive antennas (without loss of generality, we consider here the case when the number of transmit and receive antennas are equal), $\rho$ is the average signal-to-noise ratio (SNR), $\mathbf{I}$ is the $n \times n$ identity matrix, $\mathbf{H}$ is the normalized channel matrix, which is considered to be frequency independent over the signal bandwidth, and “+” denotes transpose conjugate. When the channel is random, then the capacity in (4) is random too. The expectation over the channel matrix can be employed in this case in order to define the mean (ergodic) capacity as follows [7], [13]:

$$
\langle C\rangle=\left\langle\log _{2} \operatorname{det}\left(\mathbf{I}+\frac{\rho}{n} \mathbf{H} \cdot \mathbf{H}^{+}\right)\right\rangle
$$

where $\langle C\rangle$ is the mean capacity and \langle\rangle is expectation over the channel matrix. Using Jensen's inequality and concavity of $\log$ det [10], one obtains an upper bound on the mean capacity

$$
\langle C\rangle \leq \bar{C}=\log _{2} \operatorname{det}\left[\mathbf{I}+\frac{\rho}{n} \mathbf{r}\right]
$$

where $\mathbf{r}$ is the correlation matrix with components

$$
r_{i j}=\sum_{k}\left\langle h_{i k} h_{j k} *\right\rangle, \quad i, j, k=1, \ldots, n
$$

and $h_{i j}$ denotes the components of $\mathbf{H}$. Equation (7) can be presented as follows:

$$
r_{i j}=\sqrt{\beta_{i} \beta_{j}} R_{i j}
$$

where $\beta_{i}=r_{i i}$ is the normalized average power in the $i$ th receive branch, and $R_{i j}$ are the components of the normalized correlation matrix, i.e., $\left|R_{i j}\right| \leq 1$ for any $i$ and $j$, and $R_{i i}=1$. Now we consider the effect of correlation only on the MIMO capacity. Thus, we assume that all the received average powers per receive branch are the same, $\beta_{i}=\beta$, and that $\mathbf{H}$ is normalized in such a way that $\beta=1$ (in this case, $\rho / n$ is the average SNR in each receive branch). Hence, (6) can be presented as

$$
\langle C\rangle \leq \bar{C}=\log _{2} \operatorname{det}\left[\mathbf{I}+\frac{\rho}{n} \mathbf{R}\right]
$$

where $\mathbf{R}$ is the normalized correlation matrix whose components are $R_{i j}$. In fact, the capacity $\bar{C}$ of the "average" channel provides an upper bound on the mean (ergodic) capacity $\langle C\rangle$. We further investigate this upper bound using the correlation matrix model of Section II. As detailed analysis shows, the upper bound estimated in this way is quite close to the mean capacity when all the correlation is due to the receive part of the system [5], [6] (i.e., when $\left\langle h_{i k} h_{i m} *\right\rangle=0$ for $k \neq m$ ) and when the channel is not a degenerate one [11], [6]. Thus, $\bar{C}$ may be used as a rough estimation of $\langle C\rangle$ in this case. Note that $\bar{C}$ is simple to evaluate numerically (for a given $\mathbf{R}$ ) while $\langle C\rangle$ requires lengthy Monte Carlo simulations. Due to the reciprocity of (4), the effect of transmit branch correlation can be analyzed in a similar way.

In order to estimate the mean capacity by Monte Carlo simulations, we employ some additional assumptions.

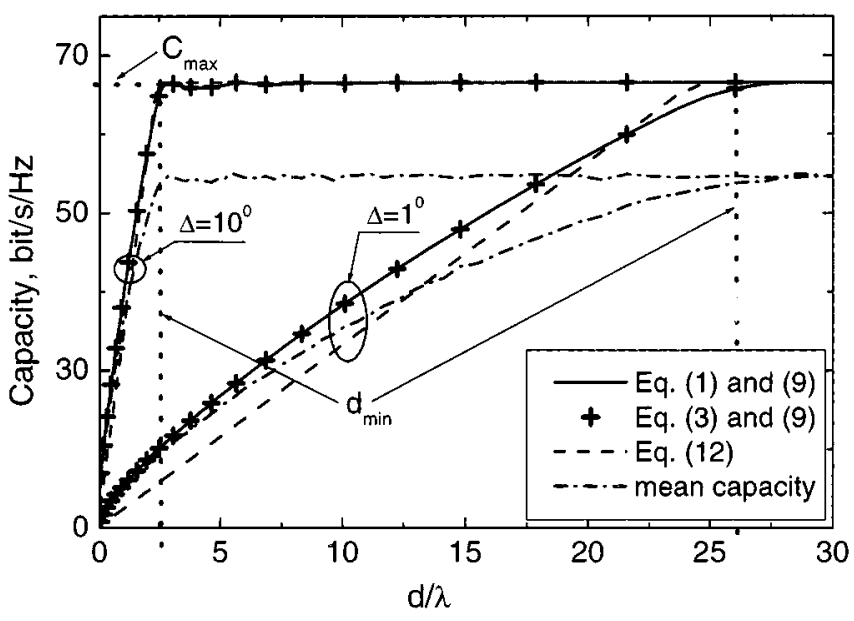

Fig. 2. MIMO capacity of the "average" channel (the upper bound) and the mean (ergodic) capacity versus $d$ for different $\Delta, N=20, n=10$ and $\rho=$ $30 \mathrm{~dB}$.

1) There are $N$ multiple paths arriving to each receive antenna from a given transmit antenna.

2) The angles-of-arrival (AOA) of these paths are uniformly distributed within $\pm \Delta$ of $\varphi$.

3) The gains of these multiple paths are i.i.d. complex Gaussian variables (i.e., Rayleigh fading) with zero mean and unit variance.

4) Each transmit antenna launches an independent set of $N$ multiple paths (i.e., independent set of AOA's and path gains) with the same statistical characteristics.

According to the assumption of independence and of equal statistical characteristics of all the transmit branches, all the terms in (7) are equal. Thus, we use (1)-(3) to evaluate $\bar{C}$ and Monte Carlo simulations to evaluate $\langle C\rangle$ for different $\Delta, \varphi$ and $d$. First, the case of $\varphi=0$ is considered (the capacity is maximum under this condition). Fig. 2 shows $\bar{C}$ and $\langle C\rangle$ versus $d$ for different $\Delta$. Note that there is good agreement between (1) and (3) when estimating the MIMO capacity. Fig. 2 indicates that the function $\bar{C}(d)$ consists of two regions: 1 ) for small $d\left(d<d_{\min }\right) \bar{C}$ increases almost linearly as $d$ increases, 2) for larger $d\left(d>d_{\min }\right) \bar{C}$ saturates and does not change significantly with $d$. Detailed analysis shows that $d_{\min }$ corresponds approximately to the first zero of $R_{i k}(d)$ for $i=k \pm 1$.

Using (3), we obtain

$$
d_{\min }=\frac{1}{2 \Delta}
$$

$C_{\max }$ is the channel capacity of an uncorrelated matrix channel,

$$
C_{\max }=n \cdot \log _{2}\left(1+\frac{\rho}{n}\right) .
$$

Further we observe that $\bar{C}(d=0)=C_{0}$ where $C_{0}=$ $\log _{2}(1+\rho)$ is the single-input single-output channel capacity (with the same total radiated power). Thus, $\bar{C}(d)$ can be approximated by the following piecewise-linear function:

$$
\bar{C}(d) \approx \min \left\{\frac{d}{d_{\min }} C_{\max }+C_{0}, \quad C_{\max }\right\} .
$$


Fig. 2 shows that (12) provides quite a good approximation when $C_{\max } \gg C_{0}$. For $\Delta>\pi / 4$ the accuracy of (3) and, consequently, of (10) degrades. In this case the following estimation is more accurate: $d_{\min } \approx 0.5$ which approximately corresponds to the first zero of (2). Thus, in a general case one may use the following estimation: $d_{\min }=\max [1 /(2 \Delta), 0.5]$. It should be noted that the mean capacity follows the same dependence on $d$ as the upper bound except that it is $15 \%$ lower in the saturation region. Thus, the upper bound provides quite an accurate estimation of the mean capacity when the effect of correlation is substantial, i.e., when $d<d_{\min }$. When $N$ increases, the maximum value of the mean capacity increases too, but by a small amount only. When $N<n$ and each transmit antenna generates the same set of AOA's, the mean capacity reduces substantially, which is in good agreement with [12]. The general dependence of $\langle C\rangle$ on $d$ shown in Fig. 2 is quite stable with respect to the assumptions i)-iv) above. For example, if the path gains are assumed to be of equal magnitudes and of independent uniformly distributed phases, the maximum capacity is less than $10 \%$ lower than that shown in Fig. 2. The same is true when each transmit antenna generates the same set of AOA's $(N \geq n)$ and the path gains are i.i.d. complex Gaussians. Of course, the assumption of full transmit branch correlation will result in a substantial capacity decrease [5], [6].

Let us now consider the case of $\varphi \neq 0$. Using an analogy with the phased array theory, one may guess that (10) should be generalized to

$$
d_{\min }=\frac{1}{2 \Delta \cos \varphi}
$$

Detailed analysis using extensive numerical simulations shows that this equation is indeed accurate provided that two constraints are satisfied

$$
\varphi<\pi / 2, \quad \Delta+\varphi \leq \pi / 2 .
$$

A general form of the function $\bar{C}(d)$ in this case is the same as in (12).

\section{CONCLUSION}

From (10), (12), and (13) we can conclude that the impact of channel correlation on capacity of a MIMO system is negligible when the two-element array beamwidth [defined as $\lambda /(d \cos \varphi)]$ is smaller than the angular spread of the incoming multipath signals. This agrees well with the results in [8].

It is also interesting to note that, as detailed Monte-Carlo analysis shows, the mean capacity follows approximately the same dependency on $d$ as in (12), with the only exception that $C_{\max }$ is 10 to $20 \%$ lower in this case, provided that the transmit branch correlation is low and the channel is not a degenerate one. Thus, the conclusion above holds for the mean capacity as well.

\section{REFERENCES}

[1] G. J. Foschini and M. J. Gans, "On limits of wireless communications in a fading environment when using multiple antennas," Wireless Personal Commun., vol. 6, no. 3, pp. 311-335, Mar. 1998.

[2] D. S. Shiu, G.. J. Foschini, M. J. Gans, and J. M. Kahn, "Fading correlation and its effect on the capacity of multielement antenna systems," IEEE Trans. Commun., vol. 48, no. 3, pp. 502-513, Mar. 2000.

[3] S. L. Loyka and J. R. Mosig, "Channel capacity of $N$-antenna BLAST architecture," Electron. Lett., vol. 36, no. 7, pp. 660-661, Mar. 2000.

[4] K. H. Li and M. A. Ingram, "Impact of clustering in statistical indoor propagation models on link capacity," in AP2000 Millennium Conf. on Antennas and Propagation, Davos, Switzerland, Apr. 9-14.

[5] S. Loyka and A. Kouki, "On the use of Jensen's inequality for MIMO channel capacity estimation," in Canadian Conf. on Electrical and Computer Engineering (CCECE 2001), Toronto, Canada, May 13-16, 2001.

[6] - "Correlation and MIMO communication architecture (invited)," in 8th Int. Symp. on Microwave and Optical Technology, Montreal, Canada, June 19-23, 2001.

[7] I. E. Telatar, "Capacity of multi-antenna Gaussian channels," AT\&T Bell Labs. Intern. Tech. Memo., June 1995.

[8] J. Salz and J. H. Winters, "Effect of fading correlation on adaptive arrays in digital mobile radio," IEEE Trans. Veh. Technol., vol. 43, pp. 1049-1057, Nov. 1994.

[9] W. C. Jakes, Jr., Microwave Mobile Communications. New York: Wiley, 1974.

[10] T. M. Cover and J. A. Thomas, Elements of Information Theory. New York: Wiley, 1991.

[11] D. Chizhik, G. J. Foschini, and R. A. Valenzuela, "Capacities of multi-element transmit and receive antennas: Correlations and keyholes," Electron. Lett., vol. 36, no. 13, pp. 1099-1100, June 22, 2000.

[12] G. G. Rayleigh and J. M. Cioffi, "Spatio-temporal coding for wireless communications," IEEE Trans. Commun., vol. 46, pp. 357-366, 1998.

[13] I. E. Telatar, "Capacity of multi-antenna Gaussian channels," Eur. Trans. Telecom., vol. 10, no. 6, Dec. 1999. 